\title{
Thrombolytic Therapy in Ischemic Strokes - A Plea for Stepping Forward from Nihilism to Optimism
}

\author{
Munakomi S* \\ Department of Neurosurgery, Nobel Teaching Hospital, \\ Biratnagar, Nepal \\ *Corresponding author: Sunil Munakomi, \\ Department of Neurosurgery, Nobel Teaching Hospital, \\ Biratnagar, Nepal
}

Received: February 03, 2017; Accepted: February 22, 2017; Published: February 24, 2017

\section{Perspective}

"Time is brain" and this notion is even more prudent when it comes to the management of ischemic infarcts. Studies have verified the role of thrombolytic therapy in improving the functional status of the patients provided it being given within 4.5 hours of insult [1] The main rational of this therapy being the rescue of the penumbra zone surrounding the infarct area thereby minimizing the number of injured brain cells.

However, when it comes to its application in the clinical scenario, even in the global front, only 1-2\% of the stroke patients are currently thrombolysed [2,3]. Despite the approval of thrombolytic therapy for the clinical use in the year 1996 from the US Food and Drug Administration (FDA), we seem to be moving in circles rather than moving forward. The major hindrance for its timely utilization would be the fear of symptomatic intra-cerebral hemorrhage leading to poor functional outcome or even mortality. However, studies have identified that the most important red flag for such complication is the presence of micro-bleeds that can be easily ruled out by Computerized Tomography (CT) imaging [4].

The Achilles heel in the timely initiation of the thrombolytic therapy is the time factor. The scenario is even more dismal when it comes to its status among the developing nations. The lack of proper pre-hospital care limits patients from reaching hospital in time. The other limitation is the right imaging modalities. Though Magnetic Resonance (MR) images with T1, T2, Diffusion Weighted Images (DWI), Apparent Diffusion Coefficient (ADC) or the penumbra sequences are ideal, studies have shown that a CT scan may just be an ideal substitute so as to minimize the time lost to treatment [5]. Lack of Stroke Unit is the other limiting issue.

In such gloomy scenario, the role of telemedicine may be a silver lining in boosting our coverage of such patients. This has effectively allowed safe allocation of patients for correct therapeutics within such narrow treatment window [6]. The outcomes from tele-therapy are in equivalent to those from the stroke team treatment [7]. Today the focus is also being given to initiate the treatment even in the ambulance. A simple cost effective, mobile data based prehospital assessment application (i-TREAT) has also been launched [8]. Mobile CT scanners, point of care labs and telemedicine application are the key pillars to the foundation that may be pivotal in increasing our reach of the patients eligible for r-TPA [9].

There is certainly some twilight in the management of ischemic stroke via thrombolytic in the developing nation as well [10]. However, to foster this initiative and not to nip in the bud of this slowly growing sub-specialty, combined and sustained help from all medical fraternities is crucial. Only then can we reach new frontiers in managing such patients in a holistic manner.

\section{References}

1. Alper BS, Malone-Moses M, McLellan JS, Prasad K, Manheimer E. Thrombolysis in acute ischaemic stroke: time for a rethink? BMJ. 2015; 350: 1075.

2. Goldstein LB. IV tPA for acute ischemic stroke: Times are changing. Neurology. 2016; 87: 2178-2179.

3. Messé SR et al. Why are acute ischemic stroke patients not receiving IV tPA? Results from a national registry. Neurology. 2016; 87: 1565-1574.

4. Raposo $\mathrm{N}$ et al. Cerebral microbleeds in acute ischemic stroke: $\mathrm{A}$ red flag for IV thrombolysis. Neurology. 2016; 87: 1526-1527.

5. Jadhav AP et al. Patient selection for stroke thrombectomy: Is CT head good enough? Neurology. 2016; 87: 242-243.

6. Madhavan M et al. Telestroke: Is it safe and effective? Neurology. 2016; 87: 145-148.

7. Rosenberg G et al. And why not thrombolysis in the ambulance (at least for some)? Neurology. 2016; 87: 214-219.

8. Chapman Smith $\mathrm{SN}$ et al. A low-cost, tablet-based option for prehospital neurologic assessment: The iTREAT Study. Neurology. 2016; 87: 19-26.

9. Barrett $\mathrm{KM}$ et al. IV thrombolysis for acute ischemic stroke: Is there a paradigm shift on the horizon? Neurology. 2016; 87: 132-133.

10. Thapa $L$ et al. Feasibility and efficacy of thrombolysis in acute ischemic stroke: A study from National Institute of Neurological and Allied Sciences, Kathmandu, Nepal. J Neurosci Rural Pract. 2016; 7: 55-60.
Austin J Neurol Disord Epilepsy - Volume 4 Issue 1 - 2017 ISSN: 2472-3711 | www.austinpublishinggroup.com Munakomi. @ All rights are reserved 